ISSN-PRINT 1794-9831 / E-ISSN 2322-7028

Vol. 18 No 2 / Mayo - Agosto, 2021 / Cúcuta, Colombia.

do) https://doi.org/10.224663/17949831.2801

Reflexión

\title{
Desarrollo de una derivación teórica de enfermería: Teoría de espiritualidad trascendental
}

\section{Development of a nursing theory derivation: Theory of transcendental spirituality Desenvolvimento de uma derivação teórica da enfermagem: Teoria da espiritualidade transcendental}

Cecilia Mazatán-Ochoa ${ }^{1}$

\section{Resumen}

La investigación en enfermería suele agregar una base teórica, que ayude a comprender la realidad de un fenómeno presente en esta disciplina; sin embargo, en ocasiones las teorías existentes, no tienen los elementos necesarios para la exploración de algunos fenómenos, por lo que se debe optar por realizar una derivación teórica que contenga los atributos necesarios para el desarrollo de una investigación. Desde este punto de vista con el presente trabajo se pretende desarrollar una derivación teórica, a partir de la teoría de la autotrascendencia, para abordar el fenómeno de la espiritualidad y la calidad de vida en el adulto mayor. Al respecto la metodología a tener en cuenta se desarrolla a partir de los aportes de Jaqueline Fawcett, quien integra cinco pasos necesarios para explicar los conceptos, proposiciones y relaciones sobre su derivación teórica. Además, se exhorta a que se tome en cuenta esta propuesta en próximas investigaciones, para que se pueda probar su efectividad.

Palabras clave: Espiritualidad, adulto mayor, enfermería, Teoría de enfermería.

\begin{abstract}
Nursing research usually adds a theoretical basis, which helps comprehend the reality of a nursing phenomenon; however, when the existent theories do not have the necessary elements for the exploration of a certain phenomenon it is possible to opt for a theoretical derivation that contains the attributes necessary for the development of a research. This work pretends to develop a theoretical derivation based on the theory of self-transcendence, to approach the phenomena of spirituality and quality of life for senior citizens. The latter under the methodology of Jaqueline Fawcett, which integrates five steps for the development of concepts, propositions, and relationships from the proposed theory derivation. Also, it encourages to use this proposal in future research to prove its efficiency.
\end{abstract}

Key words: Spirituality, Senior citizen, Nursing, Nursing theory

\section{Resumo}

A pesquisa em enfermagem geralmente contribui ao fortalecimento da base teórica, que permita compreender a realidade de um fenômeno em enfermagem, entretanto, ocasionalmente as teorias existentes, não fornecem os elementos necessários para a exploração de determinado fenômeno, pelo que pode se optar por realizar uma derivação teórica que contenha os atributos necessários para o desenvolvimento de uma pesquisa. O presente trabalho pretende

\section{Autor de correspondencia}

${ }^{1 *}$ Maestra en Enfermería. Docente. Profesora de Tiempo Completo. Universidad Autónoma de Coahuila. Piedras Negras, Coahuila. México. Correo: cecilia. mazatan@uadec.edu.mx (D) 0000-00023656-3986.

Recibido: 3 diciembre 2020

Aprobado: 10 marzo 2021

Para citar este artículo / To reference this article / Para citar este artigo:

Mazatán-Ochoa C. Desarrollo de una derivación teórica de enfermería: Teoría de espiritualidad trascendental. Rev. cienc. cuidad. 2021; 18(2):111-119. https://doi. org/10.22463/17949831.2801

(C) Universidad Francisco de Paula Santander. Este es un artículo bajo la licencia CC-BY-NC-ND 


\section{Scientific Journal of Nursing}

desenvolver uma derivação teórica, a partir da teoria da autotranscedência, para a abordagem do fenômeno da espiritualidade e da qualidade de vida no idoso. $\mathrm{O}$ anterior empregou a metodologia de Jackeline Fawcett, que integra cinco etapas para o desenvolvimento de conceitos, proposições e relações da proposta de derivação teórica. Além disso, propõe-se a implementar essa proposta em futuras pesquisas, para estabelecer a sua efetividade.

Palavras-chave: Espiritualidade, Idoso, Enfermagem, Teoria de Enfermagem.

\section{Introducción}

La investigación es una función ineludible e inseparable del profesional de enfermería (1), por eso debe realizarse de la manera más específica y segura, minimizando los errores de tipo metodológico y estadístico.

Y es aquí donde la disciplina de la Enfermería tiene una forma muy particular de realizar este abordaje de la investigación, tomando como base una teoría denominada como el conjunto organizado de conocimientos abstractos, avalados por la investigación científica y el análisis lógico, que proporcionan las bases para comprender la realidad en enfermería; además, capacitan al personal de enfermería para comprender el por qué un hecho ocurre (2). Lo que, a su vez, ayuda a que este mismo profesional trate de dar una solución a dicha problemática.

Desde esta perspectiva las teorías se obtienen a partir de la sistematización de los saberes de sus profesionales. Es por eso, que en enfermería, tienen un papel determinante en la comprensión y el análisis de los cuidados que proporciona, además de ser una herramienta útil para el razonamiento, el pensamiento crítico y la toma de decisiones necesarias para la calidad en la práctica de enfermería (3).

Sin embargo, en ocasiones el problema de investigación que se elige no concuerda totalmente con los conceptos de la teoría seleccionada; igualmente, el fenómeno que se pretende abordar, no coincide con la estructura que representan las relaciones (4) entre los conceptos, por lo que se deben construir aquellos que abarquen la medición del problema específico que se desea abordar.

Es por lo que, al seleccionar un complejo fenómeno como la espiritualidad que se entiende como una realidad que tiene que ver con la plenitud en la realización personal, evocando con ello el bienestar psicofisiológico, la búsqueda de la felicidad (5) y la calidad de vida, conocida como la percepción del individuo se orienta hacia su posición en la vida dentro del contexto cultural y el sistema de valores en el que vive, sus metas, expectativas, normas y preocupaciones que engloban su salud física, el proceso psicológico, el nivel de independencia, las relaciones sociales y las creencias personales (6) especialmente en la población adulta mayor.

De igual forma también se observa la necesidad de proponer una derivación teórica, al no encontrar una coherencia total con otras teorías. Además, es un tema que aborda desde un enfoque holístico a la persona; es decir, que se reconoce a los humanos como seres biopsicosociales y espirituales en continua relación con su entorno, lo que lo conduce a requerir un cuidado único y de calidad (7).

En consonancia con lo expuesto anteriormente se tendrá en cuenta la teoría de autotrascendencia de Pamela Reed (8), creada bajo la perspectiva de procesos de salud y desarrollo. Con esta propuesta científica se presenta a la persona adulta mayor como alguien que valora su vida, a pesar de las dificultades en las que se encuentre. Por ello, la autotrascendencia en el adulto mayor se relaciona con un sentido del todo unificado del ser adulto mayor y sus valores humanos (9).

De ahí que se estableció como objetivo desarrollar una derivación teórica, a partir de la teoría de la autotrascendencia, para abordar el fenómeno de la espiritualidad y la calidad de vida en el adulto mayor. Esta propuesta se espera que se aplique en el cuidado directo de los adultos mayores por el personal de enfermería que los asiste.

\section{Método}

Para realizar este proceso, se eligió la metodología de Jaqueline Fawcett (10), la cual consta de 5 pasos: 1. la identificación de conceptos, donde se observa el nombre del modelo conceptual; 2 . los conceptos de la teoría que comprenden las propiedades del fenómeno a estudiar y se seleccionan los conceptos de interés para 
el propio trabajo; 3. la clasificación de conceptos, que se realiza conforme a las propiedades de observabilidad y variabilidad del fenómeno, la identificación y clasificación de proposiciones, es decir, las declaraciones sobre uno o más conceptos que pretenden afirmar lo que se cree de un caso y que pueden ser relacionales o no relacionales; 4. el ordenamiento jerárquico de las propuestas, es decir, la organización de las proposiciones jerárquicamente según su nivel de abstracción, razonamiento inductivo o deductivo; $y, 5$. la construcción de diagramas, la formalización de una Estructura Conceptual-Teórico-Empírica, así como la creación de un diagrama de las relaciones de las proposiciones (Ver Tabla 1).

Tabla 1. Estructura Teórico-Conceptual-Empírica de la teoría de la espiritualidad trascendental

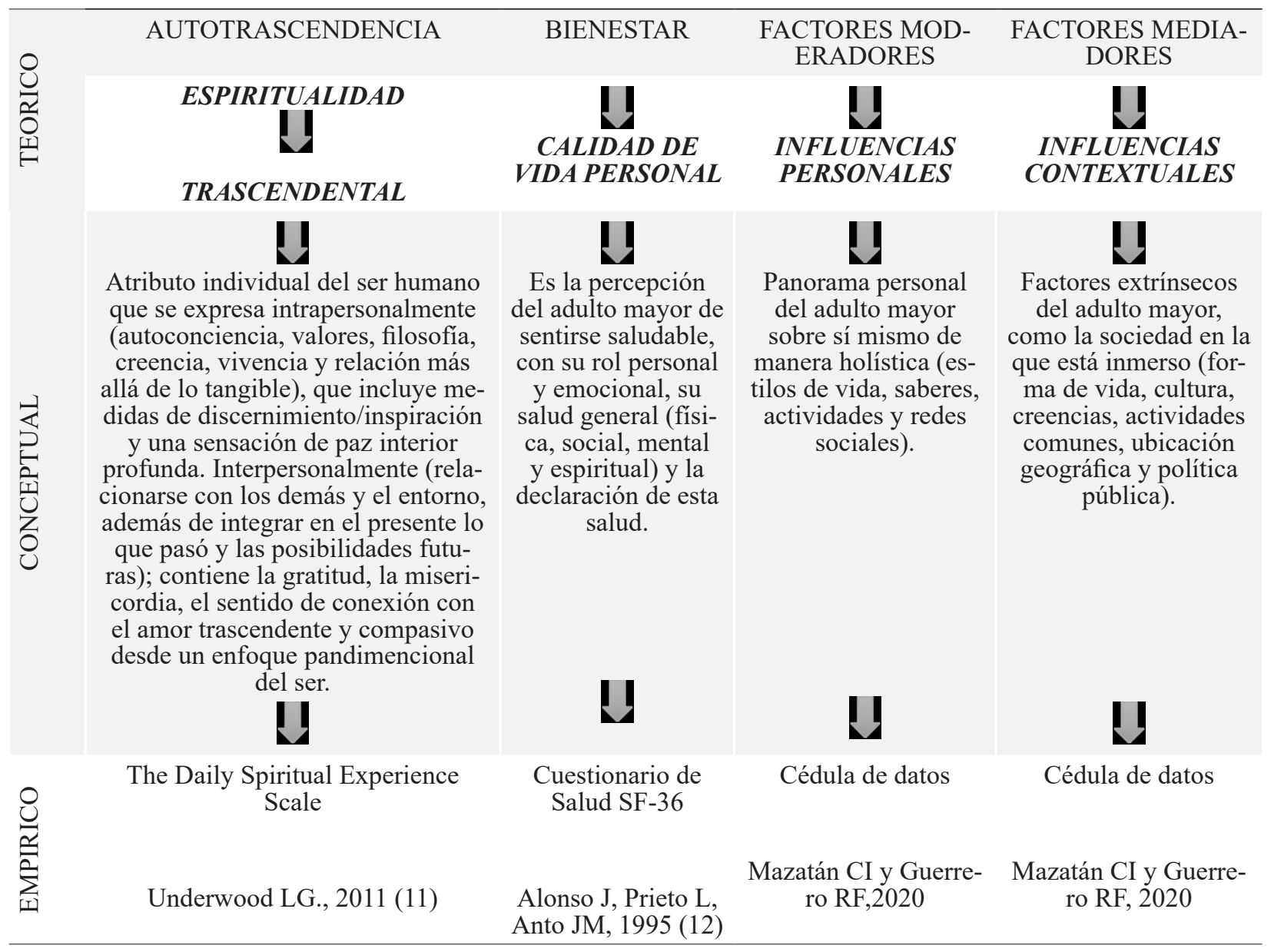

Fuente: las autoras, 2020.

\section{Desarrollo}

\section{Derivación teórica.}

\section{Paso 1. Identificación de conceptos}

Para poder derivar la teoría de "la trascendencia del yo" o "autotrascendencia" (13), se profundizó en esta, observando que tiene como propósito proveer un marco para la investigación y la práctica sobre la promoción del bienestar en medio de situaciones de vida difíciles, particularmente cuando las personas y las familias se enfrentan a la pérdida o a una enfermedad que limita la vida (14). De igual forma, se tienen en cuenta sus conceptos sobre: la autotrascendencia, la vulnerabilidad, el bienestar, los puntos de intervención, los factores mediadores y moderadores, para tomar como base algunos de ellos. 


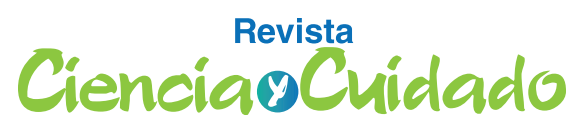

Scientific Journal of Nursing

También se revisó la fundamentación de esta teoría, la cual tuvo en cuenta la evidencia empírica relacionada con el potencial de cambio de desarrollo (influenciado menos por la edad cronológica o el paso del tiempo y más por los acontecimientos normativos y no normativos de la vida y la acumulación de experiencias de vida), que existe a lo largo de la vida, más allá de la infancia y la adolescencia, la edad adulta y los procesos de envejecimiento y muerte (15).

De la misma manera se consideraron los principios rogerianos y algunos puntos de vista filosóficos sobre la pandimensionalidad de los seres y el potencial humano de curación y bienestar. Además, se consideraron las experiencias de la práctica en la aplicación de teorías del desarrollo en la atención psiquiátrica-mental de niños y adolescentes (16).

Se debe precisar, que se construyeron dos suposiciones principales en la teoría: la primera, que los seres humanos son parte integral con sus entornos (como lo postula Rogers en la teoría de los seres humanos unitarios). Es decir, que los seres humanos son pandimensionales (17), coextensivos con su entorno y capaces de tener una conciencia que se extiende más allá de las dimensiones físicas y temporales (18). Esta conciencia puede ser experimentada a través de estados alterados de conciencia, pero más a menudo se encuentra en las prácticas cotidianas al profundizar sobre su yo y acercarse a los demás, a la naturaleza, a Dios u otras fuentes de trascendencia (16).

Respecto a la segunda suposición se parte del postulado por el cual la autotrascendencia es un imperativo de desarrollo, lo que significa que es un recurso humano que exige expresión, igual que caminar (en los niños pequeños), el razonamiento abstracto (en los adolescentes) y el duelo en aquellos que han sufrido una pérdida. Estos recursos son parte del ser humano, que debe aprovechar para obtener un mayor potencial en su bienestar. Como tal, la participación de la persona en la autotrascendencia es parte integral del bienestar, por tanto la enfermería tiene el papel de facilitar este proceso (8).

Acorde con lo expuesto anteriormente, solamente se revisaron las proposiciones que desarrolló la teoría, con las que se esclarece la perspectiva de los conceptos, con base en las relaciones entre estos (Ver Tabla 1), lo que permite mejorar la aplicación y evaluación de la teoría.

Cabe señalar que el proceso se inició de la derivación teórica, al visualizar detalladamente la teoría de Reed y observar que el fenómeno que se pretende estudiar requiere de una mayor especificidad, para la aplicación
Desarrollo de una derivación teórica de enfermería:

Teoría de espiritualidad trascendental de sus conceptos, lo que da paso a la derivación de los siguientes conceptos:

\section{Paso 2. clasificación de conceptos}

\section{Espiritualidad trascendental}

Aceptando el concepto principal de la teoría de la trascendencia del yo (autotrascendencia), Reed la define como "la capacidad de expandir los autolímites de una variedad de maneras. Intrapersonalmente (hacia una mayor conciencia de la filosofía, los valores y los sueños), interpersonalmente (para relacionarse con los demás y el entorno), temporalmente (para integrar el pasado y el futuro de una manera que tiene sentido para el presente), y transpersonalmente (para conectar con dimensiones más allá del mundo típicamente discernible)" (8).

De forma similar la autotrascendencia ha sido definida por otros autores como los niveles más altos e inclusivos o integrales de la conciencia humana, de comportarse y de relacionarse, como fines en lugar de medios, a uno mismo, a los seres humanos en general, a otras especies, a la naturaleza y al cosmos (19).

Guerrero-Castañeda la menciona como "la capacidad del ser humano para ir más allá del propio yo y como consecuencia ampliar los límites personales, mediante un camino espiritual, para dar sentido a la vida y que se enlaza con una conexión con el yo, los demás, el entorno y con el espíritu del universo; como un significado de la propia existencia" (20).

Igualmente, otros autores, definen la autotrascendencia como la creencia por la cual Dios está presente interiormente en las criaturas que evolucionan, no simplemente capacitándolas para existir, de una manera estática, sino capacitándolas para trascender lo que ya son. Dios las capacita, no sólo para ser, sino también para llegar a ser algo más de lo que son (21). También se menciona que existe relación con una cúspide en lo espiritual, lo místico o experiencias cumbre, pues la autotrascendencia busca ir más allá del yo y experimentar una comunicación más allá del yo a través de experiencias cumbres (22).

Por tanto, el concepto de autotrascendencia hace referencia a la espiritualidad trascendental que es el atributo individual del ser humano al expresarse intrapersonalmente (autoconciencia, valores, filosofía, creencia, vivencia y relación más allá de lo tangible). Además, incluye medidas de discernimiento/inspiración y una sensación de paz interior profunda que se relacionan 


\section{Cienciagecuidado}

Scientific Journal of Nursing interpersonalmente (relacionarse con los demás y el entorno, además de integrar en el presente lo que pasó y las posibilidades futuras). Igualmente, contiene la gratitud, la misericordia, el sentido de conexión con el amor trascendente y compasivo, desde un enfoque pandimencional del ser.

\section{Calidad de vida personal}

Con respecto al concepto de bienestar, Reed señala que "es una sensación de sentirse entero y saludable, de acuerdo con los propios criterios de integridad y salud" (8). Así mismo, Reyes et al., (23) lo conceptualizan como el sentir de una persona al ver satisfechas todas sus necesidades en materia fisiológica y psicológica en el presente; igualmente, hace referencia al hecho de contar con expectativas alentadoras que le sustenten su proyecto de vida en la sociedad, de acuerdo a sus experiencias como individuos que componen una comunidad; sobre todo en función de sus necesidades desde las más vitales, hasta las más superfluas, la prospectiva aspiracional y su factibilidad de realización en un lapso de tiempo admisible.

De igual forma se puede apreciar como un estado de bienestar físico, social, emocional, espiritual, intelectual y ocupacional que le permite al individuo satisfacer apropiadamente sus necesidades (24).

Es desde este punto de vista que se tomó este concepto para aplicarlo en la Calidad de vida personal y definirlo como la percepción del individuo por sentirse saludable, con su rol personal y emocional, su salud general (física, social, mental y espiritual) y la declaración de esta salud.

\section{Influencias personales e influencias contextuales}

Dicho la anterior, a continuación se tendrá en cuenta un concepto que Reed llama "factores moderadores-mediadores", a los que se refiere como "variables personales y contextuales, que pueden influir en relación existente entre vulnerabilidad y la autotrascendencia, y entre la autotrascendencia y el bienestar" (8). Sin embargo, a pesar de ser un concepto compuesto, hay quien lo define como los distintos tipos de variables intervinientes que afectan en forma diferente la relación entre otras dos variables (25).

Conviene indicar que se tomó la decisión de abordar cada uno de los factores por separado, originando dos conceptos: influencias personales e influencias contextuales.

Hay que mencionar que, la definición de Reed sobre los factores moderadores se presenta poco específica, ya que se refiere a variables personales, que pueden influir en la relación existente entre la vulnerabilidad y la autotrascendencia, y entre la autotrascendencia y el bienestar (8).

Por el contrario otros autores la han definido de forma precisa, determinando que son aquellos factores que actúan en forma previa a la interacción entre una variable independiente y una dependiente (25) o de manera semejante. Por otra parte Baron et al. (26), lo definen como las variables intervinientes que afectan la dirección y/o la fuerza de la relación entre una variable independiente y una variable dependiente (reduciéndola, incrementándola, anulándola o invirtiéndola).

\section{Paso 3. Identificación y clasificación de proposiciones}

Es así como, este concepto de factores moderadores se deriva de influencias personales como el panorama personal sobre sí mismo de manera holística (estilos de vida, saberes, actividades y redes sociales percibidas).

Se debe agregar, que según como lo señala Reed los factores mediadores, se dan de una forma inespecífica actuando durante el período de interacción de las variables dependiente e independiente, explicando el cómo y por qué se da la relación entre esas dos variables (25).

En consecuencia se decidió derivar este concepto a influencias contextuales, definiéndolo como los factores extrínsecos en el individuo como la sociedad en la que está inmerso (forma de vida, cultura, creencias, actividades comunes, ubicación geográfica y política pública).

\section{Otros conceptos}

Por otro lado, la teoría de la autotrascendencia tiene otros conceptos como la vulnerabilidad y los puntos de intervención, que no fueron seleccionados para esta derivación teórico-conceptual-empírica (Ver Tabla 2). 


\section{Scientific Journal of Nursing}

Tabla 2. Proposiciones de la teoría madre y la teoría derivada

Teoría de la Trascendencia del yo

1. El aumento de la vulnerabilidad se asocia a la trascendencia del yo. Cuando el apoyo es adecuado, la relación puede ser positiva.

2. La trascendencia del yo tiene una relación positiva con el bienestar.

3. La trascendencia del yo puede funcionar también como mediador entre la vulnerabilidad y el bienestar.

4. Los factores personales y del contexto pueden condicionar la relación entre vulnerabilidad y trascendencia del yo, y la trascendencia del yo y el bienestar.

Fuente: Pamela Reed.

\section{Paso 4. Ordenamiento jerárquico de las propuestas}

\section{Relación de conceptos de la Teoría de la Espiritual- idad Trascendental.}

Después de haber definido los conceptos de la derivación, se realizó la construcción de sus proposiciones (Ver Tabla 2), en donde se analizaron 4 conjuntos de relaciones entre los 4 conceptos que la componen, teniendo como fin, ampliar y puntualizar su aplicación, y con ello mejorar la comprensión de estas interacciones.
Teoría de la Espiritualidad Trascendental

1. El aumento de la espiritualidad trascendental repercute en el incremento de la calidad de vida y las influencias personales, con una relación positiva.

2. El incremento de la calidad de vida personal, las influencias personales y las influencias contextuales, aumentan la espiritualidad trascendental. Pero al disminuir ésta, las influencias personales y contextuales cambian a una visión negativa y la espiritualidad trascendental, puede tener una relación positiva o convertirse en negativa.

3. Las influencias personales muestran una relación positiva con la calidad de vida personal.

4. Las influencias contextuales pueden condicionar la calidad de vida y las influencias personales en una relación positiva.

\section{Primera relación}

Se inicia con la relación que analiza la espiritualidad trascendental con la calidad de vida personal y las interacciones personales. Es decir, que se describe una relación lineal y positiva, en la que se percibe una espiritualidad trascendental expresada positivamente; además, se tendrá en cuenta el que exista una buena calidad de vida personal, así como excelentes interacciones personales y viceversa.

\section{Segunda relación}

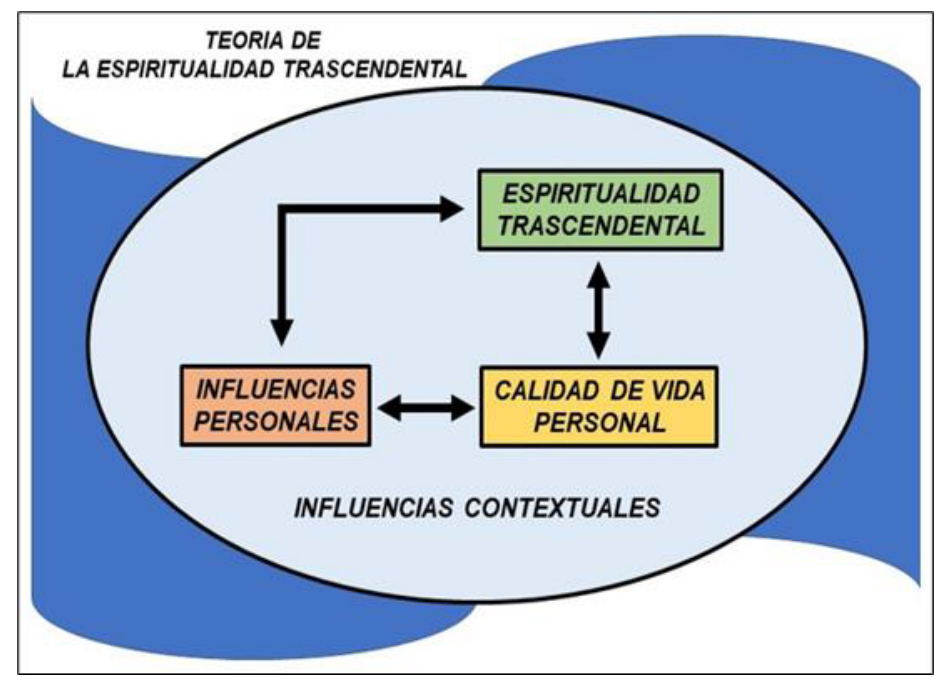

Figura 1. Diagrama de la teoría de la espiritualidad transcendental Fuente: Autora 
En segunda instancia se tendrá en cuenta la relación que integra la calidad de vida personal con las influencias personales y contextuales positivamente con la espiritualidad trascendental, mostrando una relación lineal positiva. Al contrario, una perspectiva negativa de los demás conceptos hacia la espiritualidad trascendental depende de la estabilidad o madurez frente a ésta, pues al verse influida de manera negativa, puede mantener su propia visión positiva o cambiar la dirección hacia una relación negativa.

\section{Tercera relación}

En tercer lugar, se encuentra la relación de las influencias personales que tienen una asociación lineal positiva con la calidad de vida de la persona, puesto que al mejorar las influencias personales también están presente en la calidad de vida personal y al contrario.

\section{Cuarta relación}

Para finalizar estas relaciones, se tendrán en cuenta las influencias contextuales con la calidad de vida personal y las influencias personales. Desde esta perspectiva se puede apreciar una relación lineal, positiva y unidireccional; es decir, que las influencias contextuales van a condicionar a los otros dos conceptos, puesto que no pueden interferir en las influencias contextuales.

De esta manera, el quinto paso de la derivación teórica, según la metodología de Fawcett se desarrolla en la Figura 1, con la profundización del fenómeno de la espiritualidad y la calidad de vida del adulto mayor, con respecto a la teoría de la espiritualidad trascendental.

\section{Conclusiones}

- Como resultado la estructura teórico-conceptual-empírica, sugiere un trabajo de gran importancia y complejidad, ya que, el seleccionar una teoría y trabajar con ella en la construcción del marco teórico de esta propia investigación, es necesario tener claro hacia dónde se dirige el estudio.

- En consecuencia muchas veces se pretende adaptar la propia investigación a los conceptos de una teoría, en vez de adaptar la teoría al fenómeno de estudio, lo que permite aportar a la propia disciplina la construcción de nuevos conceptos y una visión diferente a la del abordaje de determinada situación.

- Por otro lado, con este trabajo se llevó a cabo una metodología que permitió, elaborar mediante un proceso de calidad, una propuesta teórica que enriquece la profesión; por tanto, se exhorta a que se tenga en cuenta para realizar futuras investigaciones que puedan probar su efectividad.

\section{Conflicto de intereses}

La autora declara no tener conflicto de intereses, puesto que no es un estudio financiado, ni tampoco un trabajado en conjunto.

\section{Agradecimientos}

A la DCE Hilda Saray Contreras de la Fuente por compartir sus conocimientos de esta metodología y a la DCE Elizabeth Guzmán Ortiz por su orientación e impulso para realizar esta publicación.

\section{Referencias Bibliográficas}

1. Castro M; Simian D. La enfermería y la investigación. Rev. Med. Clin. Conde. 2018; 29(3): 301-310. https://doi. org/10.1016/j.rmclc.2018.04.007

2. León-Román CA. Nuevas teorías en enfermería y las precedentes de importancia histórica. Rev. Cubana Enferm [Internet]. 2017 [consultado 17 Feb 2021]; 33(4) Disponible en: http://www.revenfermeria.sld.cu/index.php/ enf/article/view/1587

3. Naranjo-Hernández Y, Jiménez-Machado N, González-Meneses L. Análisis de algunas teorías de Enfermería y su vigencia de aplicación en Cuba. Arch Méd Camagüey [Internet]. 2018 [consultado 17 Feb 2021]; 22(2): 231243. Disponible en: http://revistaamc.sld.cu/index.php/amc/article/view/5432

4. Rodríguez-Puente LA, Alonso-Castillo MM. Teoría de situación específica de autotrascendencia en dependientes del alcohol en proceso de recuperación. Aquichán [Internet]. 2015 [consultado 17 de febrero de 2021]; 16(1). Disponible en: https://aquichan.unisabana.edu.co/index.php/aquichan/article/view/5476 
5. Cornejo-Valle M, Martín-Andino Martín B, Esteso-Rubio C, Blázquez-Rodríguez M. El giro saludable: Sacrificio, sanación, Bienestar y su relación con la Espiritualidad Contemporánea. Athenea Digital. Revista De Pensamiento E Investigación Social [Internet]. 2019 [consultado 17 de febrero de 2021]; 19(2). Disponible en: https://atheneadigital.net/article/view/v19-2-cornejo

6. World Health Organization. Constitution of the World Health Organization. The first ten years of the World Health Organization [Internet]; 1958 [consultado 17 de febrero de 2021]. Disponible en: https://apps.who.int/iris/ $\underline{\text { handle/10665/37089 }}$

7. Contreras-Orozco A, Rodríguez-González K, Támara-Martínez Y. Situación de enfermería: hacia un cuidado holístico. Cultura [Internet]. 2018 [consultado 17 de febrero de 2021]; 14(2):25-4. Disponible en: https://revistas. unilibre.edu.co/index.php/cultura/article/view/4612

8. Reed PG. Theory of Self-Transcendence. En: Smith MJ, Liehr PR . Middle Range Theory for Nursing. New York: Springer Publishing Company; 2014. p. 109-139.

9. Alcocer-Sosa A, Cid-Henriquez P, Guerrero-Castañeda R. Autotrascendencia y espiritualidad en personas adultas mayores, reflexión para el cuidado. Revista Enfermería Actual [Internet]. 2021[consultado 10 Mar 2021]; (40). Disponible en: https://revistas.ucr.ac.cr/index.php/enfermeria/article/view/40800/45167

10. Fawcett J. The Relationship of Theory and Research (3rd ed). Philadelphia: F.A. Davis. 1999.

11. Underwood LG. The Daily Spiritual Experience Scale: Overview and Results. Religions. 2011; 2(1): p. 29-50. https://doi.org/10.3390/rel2010029

12. Alonso J, Prieto L, Antó JM. La versión española del SF-36 Health Survey (Cuestionario de Salud SF-36): un instrumento para la medida de los resultados clínicos. Medicina clínica. 1995; 104(20): 771-776.

13. Raile-Alligood M. Modelos y teorías en enfermería (9th ed). Barcelona: Elsevier; 2018.

14. Reed PG. The spiral path of nursing knowledge. En: Reed P, Shearer N. Nursing knowledge and theory innovation: Advancing the science of nursing practice. New York: Springer; 2011. p. 1-36.

15. Reed PG. Developmental resources and depression in the elderly. Nursing Research. 1986; 35(6):368-374.

16. Smith MJ, Liehr PR. Middle Range Theory for Nursing (3rd ed). New York: Springer Publishing Company; 2014.

17. Rogers M. A science of unitary man. En: Riehl J, Roy C. Conceptual models for nursing practice (2nd ed). New York: Appleton-Century-Crofts; 1980. p. 329-338.

18. Reed P. Nursing: The ontology of the discipline. Nursing Science Quarterly. 1997; 10(2): 76-79. https://doi. org/10.1177/089431849701000207

19. Maslow AH. The Farther Reaches of Human Nature. New York: Penguin books; 1971.

20. Guerrero-Castañeda RF, Ojeda-Vargas MG. Análisis del concepto de Autotrascendencia. Importancia en el cuidado de enfermería al adulto mayor. Cultura de los Cuidados. 2015; 19(42):26-37. http://dx.doi.org/10.14198/ cuid.2015.42.05

21. Edwards D. El Dios de la evolución: Una teología trinitaria. Santander: Sal Terrae; 2006.

22. Koltko-Rivera ME. Rediscovering the Later Version of Maslow's Hierarchy of Needs: Self-Transcendence and Opportunities for Theory, Research, and Unification. Review of General Psychology. 2006; 10(4): 302-317. 


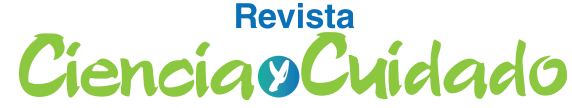

Scientific Journal of Nursing

https://doi.org/10.1037/1089-2680.10.4.302

23. Reyes-Blanco O, Franklin-Sam O. Teoría del bienestar y el óptimo de Pareto como problemas microeconómicos. CALERA [Internet]. 2016 [consultado 17 de febrero de 2021]; 14(22):50-6. Disponible en: https://www. camjol.info/index.php/CALERA/article/view/2657

24. Rubio-Olivares DY, Rivera-Martínez L, Borges-Oquendo L, González-Crespo FV. Calidad de vida en el Adulto Mayor. VERONA [Internet]. 2015 [consultado 17 de febrero de 2021];(61): 1-7. Disponible en: https://www. redalyc.org/articulo.oa? $\mathrm{id}=360643422019$

25. Kraemer HC, Wilson GT, Fairburn CG, Agras WS. Mediators and moderators of treatment effects in randomized clinical trials. Archives of general psychiatry. 2002; 59(10):877-883. https://doi.org/10.1001/archpsyc.59.10.877

26. Baron RM, Kenny DA. The Moderator-Mediator Variable Distinction in Social Psychological Research: Conceptual, Strategic, and Statistical Considerations. Journal of Personality and Social Psychology. 1986; 51(6):11731182. https://doi.org/10.1037/0022-3514.51.6.1173 